\title{
Les stratégies esthétiques dans la contestation des projets d'aménagement : le milieu géographique entre singularité et exception
}

\section{Olivier Labussière}

CIRED - Centre International de Recherche sur l'Environnement et le Développement Jardin Tropical

45 bis avenue de la Belle Gabrielle

94736 NOGENT-SUR-MARNE Cedex

E-mail : olivier.labussiere@centre-cired.fr 


\section{Résumé:}

L'essor des théories esthétiques valorisant des approches synthétiques de l'expérience et de l'action ouvre une voie nouvelle pour étudier la prise en compte du milieu en aménagement. En suivant Jacques Rancière il est possible de concevoir l'esthétique sous l'angle d'une approche contextualiste qui ne sépare pas l'intelligible du sensible. Celle-ci se révèle pertinente pour étudier la façon dont l'aménagement fait du milieu l'objet d'une discussion normée, génératrice de mésentente, qui appelle, en situation de contestation, à interroger autant le contenu du projet que les catégories cognitives qui le fondent. L'exemple de la contestation du projet de ligne très haute tension dans le Quercy Blanc, montre que la mise en œuvre de stratégies esthétiques permet d'informer l'action aménagiste mais qu'elle peine davantage à la réformer. C'est là un paradoxe intéressant de l'entrée esthétique : en valorisant la singularité du milieu géographique, elle prend le risque d'attiser une logique de l'exception qui peut bloquer le passage à l'action plutôt que d'ouvrir les voies de l'expérimentation.

\section{Mots clefs :}

Milieu géographique - aménagement - mésentente - stratégies esthétiques - singularité - exception

\section{Summary:}

The taking account of the geographical milieu in land-planing could be helped thanks to aesthetics theories based on synthetic approaches of the experience and the action. The Jacques Rancière's conception of aesthetics as the advantage of not dividing the world as perceived by the senses and by the judgement. Thus it is a meaningful approach to study how land-planning produces a misunderstanding through its way of categorizing the geographical milieu. This normative approach of the milieu leads the landplaning conflicts in a way where opponents examine both the project and its founding categories. The example of the conflict of the very high voltage transmission line project in the Quercy Blanc shows that the aesthetics strategies are able to inform the action of the landplanners but they struggle with its reframing. This point is an interesting and paradoxical one about the effectiveness of the aesthetics strategies in the land-planning conflicts: these strategies put the singularity of the geographical milieu forward but doing so there's the risk of making it an exception that could jam an innovative process of land-planning.

\section{Key-words:}

milieu - land-planning - misunderstanding - aesthetics strategies - singularity - exception 


\section{Introduction}

La prise en compte des milieux géographiques en aménagement reste encore aujourd'hui un défi méthodologique. Dans le cas des grands projets, l'évaluation environnementale peine à saisir ce qui fait la qualité d'un lieu. Ce type d'expertise a déjà fait l'objet de nombreuses remises en cause (Guigo et al., 1991 ; Passet et Theys, 1995 ; Berdoulay et Soubeyran, 1996 ; Gariépy, 1999) : il tend à segmenter ce qui est vécu comme un tout et n'aborde l'environnement du projet qu'à la marge, dans la mesure où celui-ci subit l'impact de l'infrastructure. La notion d'environnement elle-même fait l'objet d'interrogations récurrentes lorsqu'il s'agit de l'associer aux problématiques de l'action. Elle renvoie à la prise en compte de données biophysiques dans un contexte social marqué par des préoccupations gestionnaires (Tissier, 1992). De ce point de vue, le «bon usage » de l'environnement reste largement informé par le domaine de l'expertise scientifique (Larrère et Larrère, 1997). De même, en aménagement, l'évaluation environnementale reste guidée par une approche utilitariste et normative. Les éléments qui n'ont pas d'existence institutionnelle ou qui ne se prêtent pas à une mesure certaine sont difficilement pris en compte : c'est typiquement le cas de la valeur ordinaire des paysages ${ }^{1}$ dans les projets de ligne à très haute tension (THT) ou de parcs éoliens - bien que cette valeur fasse une entrée progressive et récente dans le droit (Makowiak, 2004 ; Luginbühl, 2006). Ainsi, au-delà de l'environnement, c'est la prise en compte des milieux géographiques par l'aménagement qui constitue un point d'interrogation majeur.

Cet article propose d'examiner la question esthétique comme une voie d'analyse possible des enjeux contemporains liés à l'interaction du milieu et de l'aménagement (Labussière, 2007a). C'est là une façon de renouveler le regard sur les jeux d'acteurs au cœur de ces projets : au-delà des modalités classiques de la délibération collective, suggérant une résolution des tensions sociales par des échanges argumentés, il s'agit de prêter attention aux liens des individus avec leur milieu de vie comme une ressource pour interroger les catégories cognitives de l'aménagement. En privilégiant l'entrée esthétique, nous souhaitons comprendre comment les opérations d'aménagement peuvent être informées par des données sensibles, relatives à des vécus singuliers difficilement généralisables et objectivables. Cette approche située et contextuelle est déjà repérable dans de nombreux travaux proches des questions d'aménagement sous les termes d'« environnement sensible » (Augoyard, 1995), de « vécu environnemental » (Faburel, 2005), de « géographie du sensible » (Schmitz, 2001), d' " expérience esthétique » (Balaÿ et Leroux, 2006) ou encore de "subjectivité cosmopolitique » (Blanc et Lolive, 2007). La perspective adoptée est bien celle de tenir ensemble ce qui est de l'ordre du physique, du techniquement construit, du sensible et de l'usage.

La notion de milieu, plus que celle parcellaire d'environnement, permet de relever ce défi d'une approche de synthèse. Encore faut-il en préciser le sens car la longévité séculaire de ce cadre d'approche lui doit d'avoir été traversé par les schémas explicatifs les plus divers, empruntant en géographie à l'environnementalisme comme au possibilisme radical ${ }^{2}$ (Berdoulay, 1988). Dans les années 1970, la géographie humaniste a privilégié une lecture du milieu centrée sur l'intentionnalité humaine (Relph, 1970 ; Tuan, 1976) au dépens d'un vécu dont la complexité réside aussi dans sa dimension matérielle ${ }^{3}$ (Entrikin, 1976; De Koninck, 1978). Plus récemment, Augustin Berque en a proposé une lecture plus globale : le milieu désigne autant les conditions de l'action que ses produits et la façon dont ceux-ci nous affectent en retour (Berque, 2000a). Il est à la fois empreinte et matrice. Cependant, si riche que soit ce schéma, il soulève certaines interrogations quant à la façon de penser

\footnotetext{
${ }^{1}$ La valeur ordinaire du paysage fait l'objet d'un intérêt croissant dans de nombreux travaux. Yves Luginbühl (2006) retrace la façon dont les paysages «du quotidien», «remarquables» ou «dégradés» ont récemment été pris en compte dans la Convention européenne du paysage. De son côté, Jessica Makowiak (2004) a montré comment le droit prenait en charge la question esthétique, et à travers celle-ci se saisissait de nouveaux objets comme l'enlaidissement des paysages d'entrée de ville. Une autre contribution récente, menée par Eva Bigando (2006), analyse le paysage ordinaire comme un vécu paysager auquel les habitants attribuent des valeurs en fonction de leurs systèmes de représentation; ce qui tend à montrer que le paysage ordinaire n'a pas une valeur par défaut, en contrepoint des paysages ayant une existence institutionnelle.

${ }^{2}$ L'environnementalisme valorise le rôle de l'environnement dans le fonctionnement et l'évolution des sociétés, et comprend diverses formes de déterminismes. A l'inverse, le possibilisme radical insiste sur la capacité de l'homme à se soustraire aux influences de son milieu de vie.

${ }^{3}$ S'inspirant de la phénoménologie et de l'existentialisme, l'approche humaniste s'est notamment concentrée sur l'importance des significations, des valeurs, des buts et des fins pour comprendre l'expérience humaine. Cela a contribué à mettre l'accent sur la liberté de choix de l'individu et à diminuer l'influence des causes extérieures, sociales ou physiques. De fait, l'action se déploie dans un environnement présupposé neutre, lequel n'intervient plus, comme ressource ou contrainte, que relativement aux orientations individuelles. Le risque de cette approche idéaliste de l'expérience et de l'action est d'enfermer l'individu dans un monde sans dimension concrète, inconséquent.
} 
la conduite de l'action. En particulier, sa critique de la civilisation technicienne vise à dissiper les rapports réifiant à travers lesquels nous nous saisissons de la réalité pour retrouver un rapport originel au milieu (Berque 2000b). II nous semble que la modernité peut être envisagée selon un cadre plus souple, sans opposer le milieu et la technique, à l'image de l'œuvre de Gilbert Simondon (2005). En substance, nous suivons la conception du milieu proposée par Augustin Berque, tout en souhaitant l'ouvrir davantage aux enjeux de l'action. Cette exigence paraît pouvoir être développée à l'aide des travaux de Nicholas Entrikin (1991) où la notion de milieu désigne le contexte signifiant de l'action humaine. II ne possède pas de valeur propre mais il tire sa signification des projets qui animent les hommes. Cette perspective englobante de l'expérience du sujet est essentielle à la compréhension des rapports hommes - milieu et à l'analyse des lieux singuliers qui résultent de ces rapports (Sack, 1997). Ce point de vue a le mérite de situer autant la géographie du côté de la connaissance que de l'action.

En somme, le milieu constitue un cadre de vie autant qu'un contexte d'action. La difficulté souvent rencontrée par l'aménagement est de saisir ensemble ces deux dimensions, sans instrumentaliser le tout. Le but de cet article est d'examiner en quoi la question esthétique peut nous aider à redéfinir les modalités de prise en compte du milieu en aménagement. L'originalité de l'analyse proposée est de mobiliser, à la suite de Jacques Rancière (1995), la notion de mésentente plutôt que celle de controverse. La mésentente consiste à penser, entre le milieu et l'aménagement, une relation asymétrique de conversation où ce que dit le premier n'est pas audible pour le second. Dans ce schéma, la question esthétique intervient comme un moyen dont les acteurs peuvent tirer partie pour interroger les catégories cognitives de l'aménagement et rendre sensibles certaines limites de l'évaluation environnementale.

Ce questionnement peut être utilement resitué vis-à-vis de la contestation des projets de ligne THT dans le cas français. La conflictualité grandissante qui entoure les projets à la fin des années 1980 et au début des années 1990 conduit EDF à prendre des engagements plus précis en matière d'environnement. L'invention de nouveaux pylônes « architecturés », la reconnaissance du « préjudice visuel » ou encore la résorption des «points noirs », c'est-à-dire des parties du réseau jugées inesthétiques par les riverains, sont autant de solutions proposées par le nouveau gestionnaire du réseau français, Réseau Transport Electricité (RTE). Pourtant le décalage reste patent entre ces interventions ponctuelles qui mettent l'objet technique au centre et la préoccupation esthétique qui, à travers l'expression des sensibilités, soulève une réflexion de synthèse sur les modes d'habiter. Pour aborder ces questions, nous proposons de réfléchir à partir de la contestation du projet de ligne THT 225 kV dans le Quercy Blanc. Cette contestation a duré de 1990 à 2002, date à laquelle le projet initial porté par RTE a été abandonné au profit d'un scénario alternatif - rénover les réseaux existants plutôt qu'en créer de nouveaux.

En première partie, nous montrons en quoi le renouveau des théories esthétiques - au sein desquelles nous distinguerons la pensée de Jacques Rancière - peut nous aider à problématiser la question de la prise en compte du milieu en aménagement. Ensuite, nous analysons l'influence des stratégies esthétiques dans le recadrage progressif du projet de ligne THT dans le Quercy Blanc. Enfin, l'analyse ouvre deux points de discussion relatifs à l'apport de la notion de mésentente vis-à-vis de celle de controverse dans l'étude des contestations en aménagement et à quelques effets inattendus de l'entrée esthétique en aménagement.

\section{De la mésentente à la reconnaissance du milieu : jeux d'acteurs et stratégies esthétiques}

La prise en compte du milieu en aménagement reste encore problématique, notamment en raison de méthodes d'évaluation environnementale trop sectorielles. L'essor des théories esthétiques favorisant des approches synthétiques de l'expérience et de l'action peut-il renouveler la place du milieu en aménagement ? Pour apporter des éléments de réponse à cette question, nous proposons d'examiner les théories anglo-saxonnes porteuses d'une esthétique environnementale, à la fois dans leur contenu et au travers des débats qu'elles ont suscités.

Dans le champ des théories esthétiques, un premier débat se forme autour de la notion de disinterestedness - le désintéressement comme attitude esthétique ${ }^{4}$ - au cours des années 1960.

\footnotetext{
${ }^{4}$ La notion de désintéressement est proprement kantienne. Elle renvoie, au sens strict, à l'idée que l'appréciation esthétique n'est conditionnée par aucun besoin : c'est une satisfaction obtenue sans qu'un désir en soit la cause. Précisons que ce critère (dit de « qualité ») est un des quatre qui assoient le jugement esthétique chez Kant (Sherringham, 1992). Les trois autres sont
} 
Pour se démarquer de cette posture classique, certains auteurs, à l'instar d'Arnold Berleant, soulignent les idées latentes qui l'informent : l'universalité, l'objet d'art, la contemplation, l'isolement, l'approche muséale (Berleant, 1994). Cet auteur propose une théorie de l'expérience esthétique (aesthetics of engagement) issue du pragmatisme (Dewey, 1934) et de la phénoménologie (MerleauPonty, 1976). II défend un modèle participatif fondé sur la relation réciproque entre l'environnement et l'organisme humain (Berleant, 1992) qui rencontre encore aujourd'hui une audience importante (Kupfer, 2003 ; Leddy, 2004). Si cette théorie esthétique valorise l'implication, l'action, la perception située et l'environnement, elle offre par contrecoup une focale inverse et quelque peu déformante sur l'esthétique que l'auteur nomme «classique » (dichotomie sujet / objet, contemplation passive, exclusivité de l'art, rejet du corps, absence de pensée pratique, primat de l'ordre). Cette dichotomie entre la contemplation et l'action est renforcée par un second débat entre les science-based approach et les non-science based approach (Brady, 1998). L'enjeu de celui-ci est de savoir quelle place accorder à la connaissance en général, scientifique en particulier, dans l'appréciation esthétique de l'environnement: l'approche post-positiviste d'Allen Carlson (1984), soutenant que les catégories scientifiques sont indispensables pour avoir une appréciation esthétique vraie et objective, remet à I'honneur l'activité du jugement. Cette esthétique cognitiviste, qui introduit une séparation entre l'appréciation experte et profane, nourrit un certain malaise chez les tenants d'une non-science based approach (Hepburn, 1984 ; Berleant, 1992) ; ce qui motive davantage encore la mise en quarantaine des théories esthétiques ouvertes à la question du jugement.

Cette affirmation de l'esthétique environnementale contre le modèle contemplatif s'avère quelque peu artificielle lorsqu'elle conduit à rejeter ce qui est de l'ordre du jugement et de la distanciation. D'autant plus que la multiplication des théories esthétiques affichant une posture contextualiste engage un rapport au milieu parfois discutable. Par exemple, Jean-Marie Schaeffer (2007) propose de renaturaliser le genre humain et de l'inscrire au sein d'un schéma évolutionniste global qui fait intervenir l'esthétique sur le mode stimuli / comportement. D'autres, à l'instar d'Yves Michaud (2003), critiquent l'esthétisation des modes de vie contemporains dont l'hédonisme sous-jacent pousse l'individu à satisfaire un besoin permanent d'excitation et de stimulation. Ces travaux attirent l'attention sur les dérives déterministes qui entourent certaines positions contextualistes. Comment éviter l'emballement d'une expérience esthétique qui, sous prétexte de se composer un monde, abandonne toute distance critique dans son rapport au milieu ? Pour échapper à cet écueil, la question du jugement, impliquant des actes discursifs conscients et réfléchis reste, selon nous, une dimension importante de l'expérience esthétique. Malgré les dichotomies, déjà abordées, entre une approche de l'expérience esthétique qui serait passive, à tendance objectivante, et une autre qui serait active et située, certains travaux nous incitent à revisiter ces anciens partages. En particulier la pensée de Jacques Rancière retient notre attention pour étudier les relations entre le milieu et l'aménagement, et la question esthétique au sein de ces relations.

Tout d'abord, Jacques Rancière propose une notion fondamentale : la mésentente ${ }^{5}$ (Rancière, 1995). Celle-ci constitue un cadre problématique qui met en valeur la nature asymétrique du dialogue entre l'aménagement et le milieu : le milieu peine à informer l'aménagement car la rationalité normative de celui-ci en fait un bruit, un signal non intelligible. Cette distorsion trouve, selon nous, son origine dans la constitution de l'aménagement comme science de l'action planifiée (Prost et Rioux, 1977), ce qui engage un rapport au temps marqué par la problématique de l'anticipation et un rapport à l'espace marqué par celle de l'indépendance (Soubeyran, 2007). La constitution d'un savoir scientifique de type nomothétique est le principal allié qui permet à l'aménagement de construire cet espace / temps si spécifique à la planification - mais c'est aussi à travers lui qu'il évacue la complexité de la question du milieu, compris à la fois comme le contexte contingent de l'action et comme un cadre d'existence.

La valeur ajoutée de la mésentente est de montrer qu'en faisant du milieu l'objet d'une discussion normée, l'aménagement règle la façon dont ceux qui en sont les habitants peuvent y prendre part. Cela consiste à penser qu'au-delà de la controverse sur les données particulières d'un projet (le cadrage du problème et de la solution), c'est dans la façon dont les catégories cognitives de l'aménagement font monter en représentation l'espace vécu que s'opère le partage de ce qui se prête à la discussion. Par exemple, la notion de «tracé de moindre impact » structure la planification des réseaux électriques sur la base d'une hiérarchie de valeurs génératrice de disqualification spatiale,

\footnotetext{
la « quantité » (est beau ce qui plaît universellement et sans concept), la « relation » (ce qui évoque une finalité sans fin) et la « modalité » (ce qui fait nécessité sans loi).

5 Jacques Rancière définit la mésentente de la façon suivante : « les structures de mésentente sont celles où la discussion d'un argument renvoie au litige sur l'objet de la discussion et sur la qualité de ceux qui en font un objet » (Rancière, 1995, p. 14-15).
} 
laquelle enlève à certains habitants la possibilité de faire valoir les qualités subjectives ou d'usage de leur milieu de vie. Ceci tient au fait que la mésentente instaure une hiérarchie implicite des paroles en fonction de leurs conditions d'énonciation. La mésentente ouvre donc un espace analytique à trois dimensions, comprenant l'objet de la discussion (1), la façon dont il est mis en représentation (2) et la façon dont ces représentations sont mises en circulation (3) entre les différents acteurs. Ce que Rancière appelle «partage du sensible ${ }^{6}$ est une forme particulière de déploiement (3) des représentations, qui se révèle constitutive d'un public restreint. En cela, la réflexion de l'auteur peut intéresser l'aménagement : il y a dans la façon de constituer les représentations (carte, graphique...) et de les mettre en circulation (réunion, atelier, concertation...), la génération implicite d'un public auquel ce dispositif s'adresse. Mais Rancière va plus loin, il montre que cet agencement de représentations et de réseaux dessine in fine les conditions d'entrée, comme de rejet, de formes de sensibilité et d'expérience. De ce point de vue, la mésentente est l'indexation du sensible sur l'intelligible ; c'est-à-dire l'instauration d'un mode de mise en partage (3) qui tend à réduire ce qui est en question (1) à la façon dont on le donne à sentir (2) - instillant en quelque sorte un rapport d'identité outrancier entre une réalité et sa représentation. En aménagement, le paradoxe est que certains individus peuvent à la fois être inclus, en tant qu'ils sont concernés par l'objet de la discussion (1), mais exclus parce que la façon dont on leur demande de se prononcer (3) sur la base d'une méthodologie de projet qui possède ses propres catégories et ses propres formes de représentation (2) dépouille leur parole du jeu de références grâce auquel ils habitent leur milieu. Et c'est parfois cela que vivent difficilement les opposants à un projet d'aménagement : l'irréalité de la représentation, soit l'écart entre les catégories (2) et le vécu (1). Pourtant, ce serait faire fausse route que d'assigner à la façon dont l'aménagement représente les espaces sur lesquels il intervient (2) tout le poids de cette relation asymétrique avec les acteurs et leur milieu. Ce qui est de l'ordre de la représentation peut parfois fonctionner comme un dispositif ouvert, à condition d'inventer un mode de mise en partage (3) qui assume la secondarité des formes et soit capable de mettre en suspens le pouvoir référentiel des documents graphiques. Il est essentiel de pouvoir miser de la forme pour explorer tout ce qu'un milieu vécu peut contenir comme multiplicité, pour ramifier les jeux d'acteurs jusqu'à la façon dont les individus s'attachent aux situations. L'enjeu de la mésentente porte donc sur le renouvellement de la façon dont le sensible est mis en partage (3) ; ce qui suggère de réhabiliter le lien entre les catégories (2) et le vécu (1) pour faire entendre ce que l'aménagement tient au-dehors du projet. Cette désindexation du sensible sur l'intelligible fait naître des enjeux d'ordre politique et esthétique.

D'ordinaire, la philosophie politique nous apprend que pour discuter les problèmes qui touchent à l'organisation de la vie commune, il est nécessaire de rassembler tous les individus pour que chacun se prononce. Jacques Rancière renverse le problème et part de la situation du plus exclu : comment celui-ci pourrait-il participer à la vie commune, et soulever la question de son exclusion, alors que celle-là même le tient à l'écart de la vie politique ? De ce point de vue, la politique ne peut pas fonctionner de façon cumulative car, au moment de discuter de l'organisation de la vie commune, elle laisse nécessairement de côté les exclus. II y a donc un hiatus entre la communauté des individus qui ont part à la vie politique et la question du commun. La proposition de l'auteur est de concevoir la politique non plus sur un schéma cumulatif mais transgressif. Pour que les plus exclus soient partie prenante de la vie politique, l'auteur conçoit leur mise à l'écart comme une ressource. La politique consiste alors à faire sentir ce que la société tient au-dehors d'elle-même. Ça n'est pas une stratégie consensuelle d'intégration mais une tactique de promotion d'un surplus. Cette logique excédentaire est intéressante pour penser la prise en compte du milieu en aménagement, en particulier lorsqu'un projet soulève des contestations. Disqualifié en amont - selon le schéma de la mésentente - le milieu pourrait dialoguer avec l'aménagement en jouant sur sa qualité de non-lieu. Ce non-lieu n'est pas un espace pratiqué mais ignorant des logiques de la sociabilité, à la manière de Marc Augé (1992), il désigne à proprement parler des vides, des interstices, des vacances. Comme le montre par exemple David Mangin (2004), l'aménagement urbain contemporain génère par défaut des reliquats d'espace inutilisables et inutilisés. Mais ces espaces ont une grande importance car ils sont la condition de possibilité d'une trame urbaine standard. En ce sens, le vide est une absence fondatrice. A l'instar de Rancière, pour qui la politique consiste à jouer de ces vides comme d'un potentiel pour réinterroger les pensées dont ils sont issus, nous pouvons trouver là les prémisses d'un dialogue entre le milieu et l'aménagement.

\footnotetext{
${ }^{6}$ Le partage du sensible « fixe [...] en même temps un commun partagé et des parts exclusives. Cette répartition des parts et des places se fonde sur un partage des espaces, des temps et des formes d'activité qui détermine la manière même dont un commun se prête à participation et dont les uns et les autres ont part à ce partage » (Rancière, 2000, p. 12).
} 
De ce point de vue, le milieu, c'est l'espace ouvert entre les exclus et ceux qui ont part à la vie commune. C'est l'entre-deux symbolique qui fait exister l'inégalité sociale. Néanmoins, cet entre-deux n'est pas que symbolique. II consiste aussi à jouer sur l'organisation de l'espace sensible pour forcer à penser : la politique ne sépare pas le sensible de l'intelligible. De ce point de vue, la pensée politique de l'auteur s'ouvre à une dimension géographique ${ }^{7}$. La politique n'est pas une pensée abstraite du lieu mais elle engage la relation entre les individus et leurs milieux de vie. Le milieu fait partie des ressources que l'individu peut mobiliser pour donner vie à son engagement politique. Chez Rancière la politique est ce théâtre qui intervient par effraction dans le champ social et à travers lequel la société trouve l'occasion de réfléchir sur ses apories. Le milieu n'est pas alors le décor de la vie politique, le témoignage passif d'une existence. C'est véritablement un élément actif, ressource et contrainte, qui offre virtuellement à chaque individu les conditions pour inventer une scène d'expression nouvelle. De ce point de vue, cette lecture du milieu s'accorde à notre socle conceptuel initial attentif au cadre de vie et à l'action.

Pour mieux comprendre cette prise en charge du milieu comme une ressource pour la politique et, in fine, un mode de dialogue possible avec l'aménagement, il nous faut préciser les modalités esthétiques de construction de ces scènes polémiques. D'ordinaire, les théories esthétiques renvoient à la place et au rôle de la sensibilité dans la société. Elles contribuent notamment à définir l'art comme un champ autonome de façons de faire et les modes de relations sensibles que l'appréciation des produits de l'art engage chez les individus. Rancière rompt avec cette définition traditionnelle de l'esthétique ${ }^{8}$. Celle-ci ne renvoie plus à une appréciation socialement normée des produits de l'art : esthétique est l'affirmation du sensible dans les interstices laissés par les normes sociales. Cela correspond à une logique suspensive : l'expression hors-cadre de la sensibilité sape les fondements d'un partage du sensible. L'auteur identifie deux logiques esthétiques capables de suspendre les coordonnées de la vie sociale et d'ouvrir un espace de redéfinition des règles organisant la vie commune : la logique de l'écart et celle de l'indistinction. Selon la première, les collectifs protestataires peuvent accentuer l'indisponibilité du milieu à l'égard d'un projet d'aménagement ; en suivant la seconde, ils peuvent au contraire exagérer la façon dont l'aménagement se donne le milieu comme un espace uniforme, sans qualité.

Ceci fonde ce que nous appelons des stratégies esthétiques, c'est-à-dire des processus créatifs, individuel ou collectif, consistant à transfigurer une situation de disqualification, fondée sur une normalisation du sensible, en problème public ouvrant droit à la reconnaissance d'une qualité. Ce sont donc des formes d'engagement et d'expression, à la fois politique et esthétique, permettant de remettre en discussion la place accordée au milieu en aménagement. Cela ouvre une pensée esthétique de la contestation en aménagement qui valorise une posture contextualiste compatible avec la question du jugement et de la distanciation : la relation des individus à leur milieu de vie leur permet de devenir de subtils metteurs en scène de leur condition sociale et géographique.

La contestation du projet THT en Quercy Blanc va nous permettre d'étudier les formes et la portée de ces stratégies esthétiques en aménagement. Les résultats qui suivent sont issus d'un travail de doctorat (Labussière, 2007a) dans lequel cet exemple est traité selon différentes méthodes (entretiens semi-directifs, enquête téléphonique, entretiens in situ, commentaires de planche-contacts par des photographes professionnels, réalisation à notre demande de photomontages par ordinateur).

\footnotetext{
${ }^{7}$ Lors d'une conférence, l'auteur expliquait : « la politique n'est pas la mise en œuvre d'une nature du sujet en général [...] mais la politique est création toujours de sujets spécifiques qui vont se définir par rapport à des lieux... au sens où un lieu c'est pas seulement une localité, mais c'est un espace qui va être mis en jeu, une forme d'énonciation qui est liée à la construction de cas spécifiques ». Jacques Rancière. Intervention au Colloque «Autour de Jacques Rancière, Le Maître Ignorant et l'égalité des intelligences » organisé par le CRESAL, Université de Saint-Etienne, les 29 et 30 janvier 2007.

8 L'auteur définit l'esthétique de la façon suivante: «le régime esthétique des arts est celui qui proprement identifie l'art au singulier et délie cet art de toute règle spécifique, de toute hiérarchie des sujets, des genres et des arts » (Rancière, 2000, p. 33).
} 


\section{La contestation en aménagement, une opération inventive : étude à partir du cas du Quercy Blanc}

Le cas du Quercy Blanc a retenu notre attention en raison de son exemplarité : après treize années de lutte, les opposants ont obtenu la modification du projet initial, RTE renonçant à créer une ligne THT au profit du renforcement d'une ligne existante. A l'analyse, la contestation de ce projet n'est pas apparue homogène et constante mais, au contraire, rythmée par l'apparition successive de groupes de militants animés par des stratégies de revendication différentes. Trois groupes ont été distingués: les rationalistes, les culturalistes et les légalistes. Les stratégies esthétiques ont principalement été déployées par la tendance culturaliste. Encore que les interactions progressives entre ces trois tendances, contribuant à ouvrir de nouvelles scènes de dialogue avec les aménageurs, ont favorisé leur renouvellement.

\subsection{L'émergence de stratégies esthétiques pour dire l'espace-sans-qualité}

Le projet de ligne très haute tension $225 \mathrm{kV}$ entre Cahors (Lot) et Donzac (Tarn-et-Garonne) arrive à la connaissance des élus locaux au milieu du mois de juin 1990. II vise à renforcer un réseau vétuste composé de trois lignes $63 \mathrm{kV}$ convergeant vers Cahors et dont l'économie générale serait compromise en situation de $\mathrm{N}-1$, c'est-à-dire en cas d'aléa majeur sur l'un des ouvrages. Achevé dans sa conception, le projet s'achemine vers le stade de l'enquête d'utilité publique. EDF prévoit d'ouvrir le chantier deux ans plus tard. Dans les faits, le projet n'atteindra jamais ce stade et répètera pendant treize années la procédure initiale de détermination du tracé. Le grain de sable survient au cours de l'été 1990, lorsqu'émerge l'association historique des opposants, Quercy Blanc Environnement (QBE).

Pour rassembler, les leaders de QBE s'efforcent de synthétiser les motivations hétérogènes de leurs membres dans une stratégie de protestation constructive. Leur ligne de conduite vise à associer protection environnementale et développement économique en proposant de diversifier les sources énergétiques. À cette fin, ce premier noyau d'opposants fait réaliser plusieurs contre-expertises et développe des compétences techniques spécifiques dans le domaine des moyens de production décentralisée d'électricité (cogénération, turbine à gaz). Mais cette stratégie rationaliste s'essouffle : la multiplication des études coûte cher, leur spécialisation technique échappe à beaucoup d'opposants et contre toute attente les résultats d'une des études se révèlent contradictoires. QBE en sort exsangue : son président démissionne en juin 1995. Cette première période est significative de la difficulté des opposants à faire évoluer le projet par les moyens classiques de la contre-expertise et de la représentation politique. La question qui se pose est de savoir comment cette opposition, qui rétrospectivement s'effondre à mi-parcours de sa lutte, va se renouveler et faire face à EDF, puis $\mathrm{RTE}^{9}$ pendant six années supplémentaires.

Dans le même temps, EDF progresse et atteint le stade de l'étude d'impact ${ }^{10}$. Cette étude va choquer par sa méthode analytique - pourtant classique dans ce type d'évaluation environnementale - et surtout par la façon dont EDF recense les seuls éléments de paysage ayant une existence institutionnelle. Cette étude additionne les sensibilités et les contraintes pour définir de manière arithmétique un « fuseau de moindre impact ». En fait, il ne s'agit pas d'améliorer les choix techniques du projet de façon à limiter ses «impacts » mais plutôt d'améliorer le choix du tracé de façon à diminuer le nombre de «contraintes » rencontrées. II s'agit de trouver le paysage de moindre valeur qui permettra de garantir l'insertion d'un projet standard. Cette étude d'impact, en identifiant quatorze fuseaux potentiels de passage de la ligne, aboutit au soulèvement de tout le Quercy Blanc. Les opposants découvrent qu'ils sont les habitants d'un paysage-sans-qualité : " D'un coup tout le Quercy était une zone de moindre impact ! $»^{11}$. Pour la première fois, une opposition associative se met en place à l'échelle de trois départements (Lot, Tarn-et-Garonne et Lot-et-Garonne) et se concrétise par la manifestation de Cahors, en mai 1996, réunissant 4000 à 5000 personnes.

\footnotetext{
${ }^{9}$ Nous différencions ici Electricité De France (EDF) et Réseau Transport Electricité (RTE). Ces deux instances s'occupent respectivement de la production et de la vente de l'électricité d'une part et du transport de l'électricité d'autre part. Au début de l'opposition au projet de ligne THT, en 1990, elles ne formaient encore qu'une seule et même entreprise. Depuis la loi du 10 février 2000, le réseau de transport, tout en demeurant dans EDF, est géré de manière indépendante par RTE.

${ }^{10}$ Cabinet Monbailliu et Associés. Etude d'impact. Cahors sud - Lacour. Raccordement à la ligne 225000 volts Dantou Verlhaguet. Départements du Lot et du Tarn-et-Garonne. Commanditaire EDF. 1996. 245 p.

${ }^{11}$ Entretien avec un membre de l'association QBE, le 27 mars 2006.
} 
A partir de ces événements, le collectif des opposants se recompose et diversifie ses logiques d'actions. Une nouvelle fraction (culturaliste), sans faire scission avec QBE, va néanmoins se distinguer des leaders d'alors : leur regard extérieur au jeu politique local, leur désir de mener une lutte médiatique et populaire, et leur potentiel artistique et créatif favorisent l'apparition de stratégies esthétiques. Convoquée durant les premières années sur le thème commode de la balafre, la question esthétique apparaît désormais à travers des dispositifs plus élaborés. L'exposition « Une photo pour le Quercy $»^{12}$ est une première scène d'énonciation publique qui remet la question du milieu en jeu (Labussière, 2007b) : que signifie habiter un fuseau de moindre impact ? Pour Alain Auzanneau, photographe lotois, cette exposition était l'occasion de rencontrer les personnes qui vivent sur le fuseau retenu par EDF, avec l'idée de «donner un visage au combat ${ }^{13}$. En faisant des portraits d'hommes, de visages, de mains, il s'est attaché à parler du paysage tel qu'il est vécu, travaillé, mis en forme par ceux qui l'habitent. La photographie donne à ces visages inconnus une noblesse. Elle les rend respectables. Elle humanise ce fuseau de moindre impact en rendant sensible " une histoire, une culture... ces gens là, ils étaient là avant » (Ibid.). II s'agit de donner à voir un paysage-sans-qualité comme forme de vie. Ce point de vue prend en défaut la logique réglementaire qui présidait jusque-là à l'appréciation des formes et justifiait de disqualifier l'espace sud lotois pour son moindre intérêt. Par la suite, l'opération des arbres du refus ${ }^{14}$ marque une évolution des stratégies esthétiques vers un engagement plus marqué des militants (Labussière, 2008) : les individus ont fait un usage public de leur sensibilité en plantant chez eux des arbres porteurs d'une pancarte contre la ligne THT. Ces plantations déplacent la ligne de partage entre les sphères privée et publique : les territorialités intimes deviennent le lieu d'expression d'un problème collectif. A la différence des manifestations traditionnelles qui comportent une unité de temps et de lieu, l'opération des arbres du refus a consisté en des actions différées dans le temps et pour lesquelles les individus ont agi seuls, en famille ou dans le cadre d'un groupe d'amis ou d'une association. Cela ne visait pas à magnifier leur cadre de vie, à le poser comme un objet qui force l'émerveillement, mais à rendre sensible l'épaisseur de leur quotidienneté. Les arbres du refus ont été un moyen de faire face à la disqualification spatiale, à la hiérarchie de l'exceptionnel sur l'ordinaire. Cette barrière verte, en lieu et place des pylônes, a permis de rendre sensibles les attaches subjectives que les individus entretiennent avec un espace, alors réputé par RTE, sans habitat, sans monuments, sans valeur touristique et sans patrimoine. Si l'exposition photographique a eu un impact en termes de popularisation d'une contestation, les arbres du refus ont permis d'optimiser la mobilisation d'individus éloignés géographiquement tout en développant chez eux un profond sentiment de solidarité.

Le troisième groupe, celui des légalistes, apparaît alors que le projet est relancé de façon peu habile par la préfecture du Lot, en imposant un fuseau de moindre impact parmi les 14 fuseaux possibles. Bien que faiblement en contact avec la mobilisation locale, cette nouvelle fraction d'opposants dispose de réseaux nationaux et internationaux qui lui permet de mettre en place rapidement une riposte sur le plan juridique. Leur action a joué à plusieurs niveaux : en demandant à la $C N D P^{15}$ un débat public sur l'opportunité du projet THT et en incitant les élus du conseil général du Lot à commander une expertise indépendante auprès d'Alstom. Après des efforts soutenus de 1999 à 2002, ces opposants parviennent à obtenir de la CNDP, non un « débat public » mais une préconisation de «débat local» à la charge de RTE. C'est sur cette scène que vont véritablement s'affronter, dans le fond et la forme, les visions de RTE et des opposants en matière de paysage et de cadre de vie. Ce débat local favorise la réapparition de modes de revendication valorisant une lecture esthétique du milieu: les photomontages d'André De Baere, en contrepoint de la simulation 3D de la ligne THT présentée par RTE, ont proposé une nouvelle manière de sentir : ils ont substitué à la vision aérienne et abstraite de

\footnotetext{
12 «Comment évoquer en image le Quercy d'aujourd'hui ? Quel regard poser sur les paysages, les architectures, les « ruraux » qui habitent la MAISON QUERCY au-delà des clichés sur un pays dont la beauté profonde prête à tous les effets. Au moment où de nombreux projets potentiellement dévastateurs fragilisent les espaces ruraux, comment rendre compte d'un pays en suspens, entre-deux, conscient que son avenir se joue maintenant et que la modernité n'est pas forcément technique » (Tract de présentation de l'exposition « Une image pour le Quercy », 1997). Cette citation montre combien la réflexion portée autour du paysage ne cède pas à la facilité d'un discours construit sur la dichotomie du beau et du laid. Le débat paysager s'émancipe d'une stricte appréciation picturale et pose la question de la façon dont les populations locales habitent la « Maison Quercy ».

13 Entretien avec Alain Auzanneau, le 12 avril 2006.

14 Cette opération, initiée en 1999 et achevée en 2002, consiste à planter 115 arbres en lieu et place des 115 pylônes prévus sur le fuseau 2B retenu par Réseau transport électricité (RTE).

15 Héritière de la circulaire «Bianco» et des procédures québécoises du «bureau des audiences publiques sur l'environnement »(BAPE), la CNDP a été instaurée le 4 septembre 1997 par Dominique Voynet. Dans le cas des réseaux de transport d'électricité, cette instance est chargée d'ouvrir, d'une part, une concertation pour tout projet de ligne dont la tension est supérieure ou égale à $400 \mathrm{kV}$ et la longueur supérieure à 10 kilomètres, et de l'envisager, d'autre part, pour les projets dont la tension est supérieure ou égale à 200 kV et la longueur supérieure à 15 kilomètres.
} 
la simulation numérique, le point de vue de l'expérience sensible, celle du pèlerin, du marcheur, du paysan. (Labussière et De Baere, 2007).

\subsection{De la reconnaissance du milieu au blocage de l'action aménagiste}

Les stratégies esthétiques, outre leur impact médiatique, ont contribué au recadrage du projet sur plusieurs points. Tout d'abord, elles ont renforcé les solidarités entre des groupes d'opposants aux lignes de conduites hétérogènes, certains ne parvenant pas à suivre la stratégie rationaliste fondée sur la contre-expertise et la spécialisation technique. Elles ont aussi offert des solutions inédites de gestion de l'effort de contestation dans l'espace et dans le temps, à l'image de l'opération des arbres du refus qui permit, dans un espace rural aux habitations dispersés, de rassembler malgré les contraintes de lassitude et d'isolement. Elles ont encore donné aux opposants les moyens de rediscuter le statut d'espace-sans-qualité attribué par RTE au Quercy Blanc. L'exposition photographique, les arbres du refus, les photomontages ont redonné une voix à un espace réputé sans qualité. Sans crispation identitaire ni sublimation outrancière, ces stratégies esthétiques ont démontré que les formes géographiques n'avaient pas une signification conventionnelle dont on pouvait déduire abstraitement la grandeur ou l'insignifiance. En valorisant le point de vue de l'expérience sensible, elles ont souligné que le milieu constituait un surplus ignoré de l'évaluation environnementale de RTE.

A l'issue de ce débat local, RTE justifie son retrait du Quercy Blanc notamment pour des raisons environnementales : "nous constatons qu'il faut distinguer la notion de moindre impact en site vierge et la solution offerte par les solutions alternatives de l'étude Alstom, dont le but consiste à minorer l'impact en utilisant un couloir de lignes existant $»^{16}$. L'invention terminologique de " moindre impact en site vierge » est une petite révolution dans le domaine de la planification des réseaux électriques. D'ordinaire la notion de moindre impact contribue à la justification d'un projet en identifiant des espaces de faibles contraintes, et donc de moindre intérêt. Mais parler de « site vierge » consiste à reconnaître que même un espace de moindre contrainte n'est pas sans qualité. C'est véritablement la reconnaissance du Quercy Blanc comme milieu de vie singulier. "Là, on s'est trouvé avec une contradiction que les zones les moins habitées se sont trouvées les plus valorisées [...] le paysage vierge qui est reconnu comme étant plus valorisant ou à valoriser au moins autant qu'un paysage lié à des monuments historiques... ça, ça a été un fait nouveau. Ça fera certainement école » ${ }^{17}$.

La reconnaissance par RTE de la singularité du Quercy Blanc est explicite. Pourtant, RTE n'adapte pas sa méthodologie de projet à cette lecture plus complexe du milieu - par exemple, en adaptant son modèle d'action par le recours à l'enfouissement des réseaux. La décision du gestionnaire du réseau est d'intervenir sur un autre espace, en renforçant l'axe nord existant. Pourquoi cette délocalisation ? L'abandon du projet $225 \mathrm{kV}$ à travers le Quercy Blanc est a priori évident : RTE a, dès la fin du débat local, expliqué qu'il ne pouvait assumer le surcoût dû à l'enfouissement. Pourtant, cette justification économique n'a pas qu'une signification locale, elle trouve aussi un sens à l'échelle nationale. Le Quercy Blanc constituait un cas de jurisprudence que RTE n'a pas souhaité voir aboutir, comme nous l'expliqua le chef du projet Cahors : "S'il faut mettre tout en souterrain et que la puissance publique nous dit: Messieurs, allez-y, payez quatre ou cinq fois plus cher dans une zone [le Quercy Blanc] que... si on le fait là, on va devoir le faire partout en France... et on n'a pas les moyens de le faire $»^{18}$.

RTE souhaite éviter de constituer un précédent en matière d'enfouissement des réseaux $225 \mathrm{kV}$ en réalisant un projet d'exception dans une zone initialement retenue pour son moindre intérêt environnemental. Et il ne serait pas nécessaire que le cas du Quercy Blanc entre dans le droit pour faire jurisprudence. Les collectifs anti-THT en France connaissent généralement bien les situations qui ont donné raison aux opposants et demandent à RTE que l'histoire se répète ${ }^{19}$.

\footnotetext{
${ }^{16}$ Les deux autres raisons tiennent à la volonté des élus et des populations locales de mettre en place une Maîtrise de la demande d'électricité (MDE) et au surcoût de l'enfouissement de la ligne THT que suppose le passage dans le Quercy Blanc par rapport à l'aérien (Compte-rendu intégral. Débat local sur le renforcement de l'alimentation électrique du Lot. Mardi 17 décembre 2002. Espace Valentré à Cahors, 18h00 - 22h00. Réunion générale de clôture).

${ }^{17}$ Entretien avec l'ingénieur en communication de RTE pour le projet Cahors, le 24 avril 2006.

${ }^{18}$ Entretien avec le chef du projet Cahors de RTE, le 24 avril 2006.

${ }^{19}$ A titre d'exemple, les opposants au projet THT en Quercy Blanc demandaient un nouveau «Louron », après l'abandon du projet THT, en 1996, dans cette vallée pyrénéenne.
} 
Ce cas d'étude fait émerger un paradoxe intéressant : sorti de la mésentente, le milieu est désormais perçu par le gestionnaire du réseau de transport d'électricité mais il ne devient pas pour autant moteur de son action. Voilà un potentiel que les aménageurs refusent d'actualiser. La crainte de voir une expérimentation monter en généralité et remettre en cause un modèle dominant de pratique aménagiste, se traduit par une attitude conservatrice : RTE préfère trouver un milieu plus approprié à son modèle d'action plutôt que d'adapter ce dernier à la complexité croissante du milieu initial. Cet exemple appelle à approfondir les enjeux soulevés par l'entrée esthétique : si celle-ci peut constituer un terrain d'entente entre le milieu et l'aménagement, le défi qui la sous-tend dépasse l'idée de promotion sensible du milieu et pose le problème d'une prospective qui s'accorde avec l'invention de nouveaux modèles d'action.

\section{Intérêts et effets inattendus de l'entrée esthétique en aménagement}

La question esthétique offre une lecture originale des enjeux liés à la prise en compte du milieu en aménagement. L'analyse qui précède ouvre deux points de discussion relatifs à l'apport de la notion de mésentente vis-à-vis de celle de controverse dans l'étude des contestations en aménagement et à quelques effets inattendus de l'entrée esthétique en aménagement.

\section{i. La mésentente, un nouveau regard sur la contestation en aménagement}

II nous semble important de revenir sur l'apport de la notion de mésentente. Celle-ci peut élargir l'étude des contestations en aménagement en soulevant des préoccupations que ne paraît pas pouvoir saisir la notion de controverse. Cette dernière désigne, à la suite des travaux de sociologie des sciences et techniques qui la fondent (Callon, 1986), une situation de débat social et politique autour de connaissances scientifiques ou techniques mal assurées: elle prend alors l'acception de «puissant dispositif d'exploration et d'apprentissage des mondes possibles » (Callon, Lascoumes et Barthe, 2001, p. 50). La controverse tire son efficacité de la démocratisation de l'expertise et plus généralement de la capacité d'individus à s'instituer porte-parole d'humains ou de non-humains. Le paradoxe de cette ouverture est qu'elle repose sur la diffusion de régimes d'expression pour le moins codés : la preuve scientifique et la justification politique. Or ces régimes reposent sur des règles occasionnant certains impensés : ils présupposent une situation égalitaire de conversation, même si les individus ont des ressources et des capacités souvent insuffisantes pour se faire entendre ; ils ne rendent partageables et recevables les paroles qui empruntent ces régimes qu'à condition de résultats et de causes suffisamment générales.

La notion de mésentente dépasse le débat autour de l'objet de la discussion pour tenir compte de la façon dont cet objet se prête à discussion. C'est le cas en aménagement où le milieu désigne à la fois ce dont il est question et les conditions d'énonciation de ceux qui, l'habitant, souhaitent se faire entendre. Peu de théories de l'aménagement se soucient des enjeux relatifs à cette normalisation du sensible, et à ses implications en matière de débat public et de conduite de l'action. Par exemple, le courant anglo-saxon de la mobilisation sociale (social mobilization) a, par le passé, conçu l'aménageur comme le gestionnaire d'un contexte social de communication, marqué par de multiples sources de désinformation, dont le but était de favoriser une interaction sociale rationnelle (Forester, 1989 ; 1999). Mais cette forme de pragmatisme communicationnel proche des options théoriques d'Habermas ne permet pas de déceler une situation de mésentente. A travers cette dernière, l'enjeu n'est pas de faire entendre de nouveaux arguments mais de démontrer qu'il existe un lien entre les catégories débattues (zone de moindre impact) et les modes d'existence qu'elles abritent. Par conséquent, l'espace de la négociation ne peut se restreindre à la rationalité afférente à une catégorie cognitive (délimiter la « bonne » zone de moindre impact), il inclut aussi des ramifications vers l'espace vécu, que la non prise en compte transforme en scène sensible et polémique.

Ainsi, l'apport de la mésentente est de montrer que les débats entre aménageurs et opposants ne peuvent se régler sur la base d'une stricte rationalité argumentative. La confrontation des arguments excède leur seul contenu: c'est une forme d'expérience qui doit s'accorder avec les conditions d'existence des individus. La mésentente désigne une situation où le milieu se manifeste comme le dehors de la pensée aménagiste - ce que peuvent illustrer les citations ${ }^{20}$ ci-après :

\footnotetext{
${ }^{20}$ Compte-rendu intégral. Débat local sur le renforcement de l'alimentation électrique du Lot. Jeudi 29 novembre 2002. Salle des fêtes à Montdoumerc, 18 h00 - 21h00. Thématique « Economie ».
} 
- RTE : « Je ne vois pas comment, d'une façon rationnelle, on va intégrer ce que vous semblez vouloir prendre en compte au niveau des coûts de l'environnement sur la $225000 \mathrm{~V}$ et son impact. Certains éléments sont chiffrés au niveau du protocole État-EDF-RTE et sont intégrés dedans. Ils prennent en compte l'environnement, mais pas de la manière dont vous le souhaitez ».

- Public: "Pour moi, il n'y a pas de coût d'indemnisation qui tienne. Il y a bien sûr l'environnement, je suis entièrement d'accord, mais il y a aussi des gens qui y vivent, dans cet environnement ».

Lors du débat local, l'enjeu environnemental est audible pour RTE parce qu'il est mesurable, chiffrable et qu'il peut faire l'objet de négociations avec les particuliers (ajustement à la marge du projet, pylônes esthétiques, indemnisations). En revanche, la question du milieu tombe dans l'espace de la mésentente. A l'image de l'échange précédent, RTE entend « environnement » lorsque les opposants parlent du Quercy Blanc comme d'un milieu de vie. Et lorsque les opposants refusent des indemnisations relatives à l'« environnement », RTE se retrouve face à un surplus inclassable.

Le défi esthétique en aménagement ne se limite donc pas à la prise en compte des sensibilités ; il appelle une réflexion plus globale sur la façon dont ces sensibilités, en confrontant des modes d'existence à des catégories cognitives censées les qualifier, les intégrer ou les ignorer, constituent un plan d'épreuve pour l'action aménagiste. Ainsi, la question du sensible n'intervient plus en aval, comme un critère à adjoindre aux démarches de projet existantes, elle fait partie d'une problématique qui croise en amont la géographie et l'aménagement.

ii. L'entrée esthétique en aménagement : le milieu géographique entre singularité et exception

Du cas étudié ressort une interférence intéressante entre la problématique esthétique et celle de la jurisprudence - au sens d'un cas pouvant constituer un précédent en matière de prise en compte du milieu dans la planification des lignes THT. Alors que l'entrée esthétique fait de la singularité du Quercy Blanc un cadre invitant à repenser l'action, celle de la jurisprudence conduit au contraire à y voir une exception a fort effet inhibant. Par quel procédé le milieu en vient-il ainsi à changer de statut?

En première analyse, ne pourrions-nous pas déceler, derrière l'argument recevable du surcoût lié à l'enfouissement des réseaux THT, la présence d'une rhétorique réactionnaire ? C'est ce que suggère l'analyse par Albert Hirschman (1991) de l'argument dit de la «mise en péril »: le changement, souhaitable en principe, entraîne des coûts ou des conséquences inacceptables. Telle est bien la posture de RTE dans notre cas d'étude : reconnaître la singularité d'un milieu est juste, agir en conséquence n'est pas raisonnable.

Hirschman approfondit ce procédé par un renvoi à l'analyse antérieure de Cornford sur le "principe du dangereux précédent »: « [lequel] commande de s'abstenir d'accomplir aujourd'hui un acte que l'on sait être juste de crainte de manquer du courage nécessaire pour en faire autant demain dans un cas qui, pour différer fondamentalement de la situation présente, n'en présente pas moins avec elle une ressemblance superficielle ${ }^{21}$. Cette définition apporte un éclairage supplémentaire sur le paradoxe auquel se confronte l'entrée esthétique dans les cas de contestation de projets d'aménagement. L'argument du dangereux précédent réduit la logique du surplus, fondatrice des stratégies esthétiques chez Rancière, à la situation plus classique de l'exception. Le surplus manifeste un au-delà de la règle. L'exception la présuppose. Plus encore, elle la confirme dès lors qu'elle s'accompagne d'un jugement par analogie. C'est là le ressort si puissant dont nous fait part Cornford : ce qui retient le passage à l'action c'est cette « ressemblance superficielle » établie entre un cas présent et un cas à venir. Cette équivalence, qui ne peut être admise qu'à titre spéculatif, parvient à généraliser ce statut d'exception et à en faire une contrainte majeure pour l'action.

On peut difficilement séparer cette analyse sur la singularité du lieu et le blocage de l'action aménagiste, sans la rapporter à l'architecture centralisée du modèle français de production d'électricité et à la gestion de ses réseaux. De ce fait, la possibilité de croiser les référentiels du milieu et de l'aménagement est plus difficile - ce qui, inversement, paraît davantage envisageable dans le

\footnotetext{
${ }^{21}$ Au sujet de F.M. Cornford, Hirschman renvoie à son écrit de 1908, « Microcosmographia ». Cambridge : Bowes and Bowes.
} 
cas d'unités décentralisées de production d'électricité comme l'éolien (Nadaï et Labussière, à paraître).

\section{Conclusion}

L'essor des théories esthétiques valorisant des approches synthétiques de l'expérience et de l'action ouvre une voie nouvelle pour étudier la prise en compte du milieu en aménagement. En suivant Jacques Rancière il est possible de concevoir l'esthétique sous l'angle d'une approche contextualiste qui ne sépare pas l'intelligible du sensible. Celle-ci se révèle pertinente pour étudier la façon dont l'aménagement fait du milieu l'objet d'une discussion normée, génératrice de mésentente, qui appelle, en situation de contestation, à interroger autant le contenu du projet que les catégories cognitives qui le fondent.

De ce point de vue, la notion de mésentente élargit l'étude des contestations en aménagement aux stratégies esthétiques, comprises comme des processus créatifs, individuel ou collectif, consistant à transfigurer une situation de disqualification, fondée sur une normalisation du sensible, en problème public ouvrant droit à la reconnaissance d'une qualité. Ceci permet de dépasser l'analyse quelque fois trop mécaniste des revendications esthétiques, présentée comme un réflexe de crispation territoriale autour d'attributs identitaires individuels et collectifs. Dans le cas étudié, les stratégies esthétiques ne constituent pas une résistance ou un ressort qui répondrait à une pression venue du dehors. Elles sont l'affirmation d'une vie sensible absente de la représentation que donne l'aménagement d'un environnement intelligible et maîtrisable.

L'intérêt de l'entrée esthétique est également de mettre en avant certains points d'apprentissage et de blocage relatifs à la prise en compte du milieu en aménagement. Le cas étudié a montré qu'une lecture esthétique du milieu contribuait à informer l'action, mais qu'elle peinait davantage à la réformer. Une analyse approfondie a permis de mettre en avant la traduction opérée par l'aménagement entre la logique du surplus, motivant la reconnaissance du Quercy Blanc comme « zone de moindre impact en site vierge », et celle de l'exception, justifiant le blocage de l'action par l'argument du « dangereux précédent ». Ceci témoigne de la difficulté à penser en aménagement le renouvellement des logiques d'action à partir d'une lecture attentive à ce qui fait la qualité d'un lieu pour ses habitants. Ce constat appelle à travailler sur les paramètres autant théorique, pratique qu'institutionnel qui permettraient de libérer l'action aménagiste d'une logique de l'exception, pour rouvrir les voies de l'expérimentation.

\section{Indications bibliographiques}

Augé Marc (1992). Non-lieux. Introduction à une anthropologie de la surmodernité. Paris : Seuil, 150 p.

Augoyard Jean-François (1995). L'environnement sensible et les ambiances architecturales. L'Espace géographique, $\mathrm{n}^{\circ} 4$, pp. 302-319.

Balaÿ Olivier et Leroux Martine (2006). L'expérience esthétique de l'autoroute A47 entre Givors et Saint-Etienne. Rapport de recherche financé par la DDE 42, CRESSON, $123 \mathrm{p}$.

Berdoulay Vincent (1988). Des mots et des lieux. La dynamique du discours géographique. Paris : Éditions du CNRS, $106 \mathrm{p}$.

Berdoulay Vincent et Soubeyran Olivier (1996). Débat public et développement durable. Expériences Nord-Américaines. Paris : Éditions Villes et Territoires, $156 \mathrm{p}$.

Berleant Arnold (1992). The aesthetics of the environment. Philadelphia: Temple University Press, 218 p.

Berleant Arnold (1994). Beyond disinterestedness. The British Journal of Aesthetics, vol. 34, $\mathrm{n}^{\circ}$ 3, pp. 242-254.

Berque Augustin (2000a). Ecoumène. Introduction à l'étude des milieux humains. Paris : Belin, $271 \mathrm{p}$.

Berque Augustin (dir.) (2000b). Logique du lieu et dépassement de la modernité. Tomes 1 et 2. Ousia : Librairie philosophique Vrin, 390 p. et 294 p.

Bigando Eva (2006). La sensibilité au paysage ordinaire des habitants de la grande périphérie bordelaise (commune du Médoc et de la Basse Vallée de l'Isle) ; sous la dir. de Guy Di Méo. Thèse de doctorat : Géographie : Université Bordeaux 3, $490 \mathrm{p}$. 
Blanc Nathalie et Lolive Jacques (2007). Les subjectivités cosmopolitiques et la question esthétique, in L'émergence des cosmopolitiques; sous la dir. de Jacques Lolive et d'Olivier Soubeyran. Paris : Éditions La Découverte, pp. 352-382.

Brady Emily (1998). Don't eat the daisies: disinterestedness and the situated aesthetic. Environmental values, vol. $7, \mathrm{n}^{\circ} 1$, pp. 97-114.

Callon Michel (1986). Éléments pour une sociologie de la traduction. La domestication des coquilles Saint-Jacques et des marins pêcheurs dans la baie de Saint-Brieuc. L'année sociologique, vol. 36, pp. 169-208.

Callon Michel, Lascoumes Pierre et Barthe Yannick (2001). Agir dans un monde incertain. Paris : Seuil, $358 \mathrm{p}$.

Carlson Allen (1984). Nature and positive aesthetics. Environmental ethics, $\mathrm{n}^{\circ} 6$, pp. 5-34.

De Koninck Rodolphe (1978). Contre l'idéalisme en géographie. Cahiers de géographie du Québec, vol. 22, n 56, pp. 123-145.

Dewey John (1934). Art as experience. New York: Capricorn Books, 355 p.

Entrikin John Nicholas (1976). Contemporary humanism in geography. Annals of the Association of American Geographers, vol. 66, $\mathrm{n}^{\circ}$ 4, pp. 615-632.

Entrikin John Nicholas (1991). The betweeness of place. Towards a geography of Modernity. Baltimore : The John Hopkins University Press, $196 \mathrm{p}$.

Forester John (1989). Planning in the face of power. Berkeley: University of California Press, $283 \mathrm{p}$.

Forester John (1999). The deliberative practitioner. Encouraging participatory planning processes. Cambridge: The MIT Press, $305 \mathrm{p}$.

Faburel Guillaume (2005). Le vécu environnemental comme objet d'action publique. Des méthodes d'évaluation de la gêne sonore pour une autre implication des populations dans les débats. Communication au Colloque scientifique sur les observatoires socio-économiques et environnementaux d'infrastructures linéaires, SETRA, 17-18 mars 2005, 15 p.

Gariépy Michel (1999). L'analyse de paysage au sein de l'évaluation environnementale ou l'aménagement à l'ère de la rectitude politique, in Le paysage, territoire d'intentions; sous la dir. de Philippe Poullaec-Gonidec, de Michel Gariépy et de Bernard Lassus. Paris: L'Harmattan, pp. 95-113.

Guigo Maryse et al. (1991). Gestion de l'environnement et études d'impact. Paris : Masson, 231 p.

Hepburn Ronald (1984). Contemporary aesthetics and the neglect of natural beauty, in Wonder and other essays. Edimburgh: Edimburgh Universtiy Press, $183 \mathrm{p}$.

Hirschman Albert (1991) Deux siècles de rhétorique réactionnaire. Paris: Fayard, $294 \mathrm{p}$.

Kupfer Joseph (2003). Engaging nature aesthetically. Journal of aesthetic education, vol. 37, $\mathrm{n}^{\circ} \mathrm{1}$, pp. 77-89.

Labussière Olivier (2007a). Le défi esthétique en aménagement: vers une prospective du milieu. Le cas des lignes très haute tension (Lot) et des parcs éoliens (Aveyron et Aude); sous la dir. de Vincent Berdoulay: Thèse de doctorat : géographie et aménagement : Université de Pau et des Pays de l'Adour, $607 \mathrm{p}$.

Labussière Olivier (2007b). La valeur ordinaire du paysage: stratégies photographiques des opposants à la ligne très haute tension en Quercy Blanc, in Résister à la disqualification sociale. Espaces et identités ; sous la dir. de Michel Koebel et d'Emmanuelle Walter. Paris : L'Harmattan, pp. 161-186.

Labussière Olivier et De Baere André (2007). Entre « insertion » et « mise en situation » paysagère : la mésentente au cœur d'un projet de ligne très haute tension. Cosmopolitiques, $\mathrm{n}^{\circ} 15, \mathrm{pp}$. 95-106.

Labussière Olivier (2008). Géographie du sujet et construction des territorialités. Le cas des arbres du refus contre la ligne très haute tension en Quercy Blanc, in Géodoc $n^{\circ} 55$ : « Mobiliser la notion d'acteur en géographie »; sous la coord. de Fabienne Cavailé et de Johan Milian, pp. 53-65.

Larrère Catherine et Larrère Raphaël (1997). Du bon usage de la nature. Pour une philosophie de l'environnement. Paris : Alto Aubier, $355 \mathrm{p}$.

Leddy Thomas (2004). The nature of everyday aesthetics, in The aesthetics of everyday life; sous la dir. d'Andrew Light et de Jonathan Smith. New York: Columbia University Press, pp. 3-22.

Luginbühl Yves (dir.) (2006). Paysage et bien-être individuel et social, in Paysage et développement durable : les enjeux de la Convention européenne du paysage. Strasbourg: Éditions du Conseil de l'Europe, pp. 31-55.

Makowiak Jessica (2004). Esthétique et droit. Paris : LGDJ, 403 p.

Mangin David (2004). La ville franchisée. Formes et structures de la ville contemporaine. Paris: Éditions de la Villette, 398 p. 
Merleau-Ponty Maurice (1976). Phénoménologie de la perception. Paris: Gallimard, 531 p.

Michaud Yves (2003). L'art à l'état gazeux. Essai sur le triomphe de l'esthétique. Paris: Editions Stock, $204 \mathrm{p}$.

Nadaï Alain et Labussière Olivier (à paraître). Windpower planning in France (Aveyron). From state regulation to local planning. Land Use Policy.

Passet René et Theys Jacques (1995). Héritiers du futur. Aménagement du territoire, environnement et développement durable. La Tour d'Aigues : DATAR ; Éditions de l'Aube, 224 p.

Prost Robert et Rioux Laval (1977). La planification. Montréal : Les Presses Universitaires du Québec, $129 \mathrm{p}$.

Rancière Jacques (1995). La mésentente. Politique et philosophie. Paris : Galilée, 188 p.

Rancière Jacques (2000). Le partage du sensible. Esthétique et politique. Paris : La fabrique éditions, $75 \mathrm{p}$.

Relph Edward (1970). An inquiry into the relations between phenomenology and geography. The Canadian Geographer, vol. 14, n 3, pp. 193-201.

Sack Robert (1997). Homo Geographicus. Baltimore: Johns Hopkins University Press, 292 p.

Schaeffer Jean-Marie (2007). La fin de l'exception humaine. Paris : Gallimard, 464 p.

Schmitz Serge (2001). La recherche de l'environnement pertinent. Contribution à une géographie du sensible. L'Espace géographique, vol. 30, n 4, pp. 321-332.

Sherringham Marc (1992). Introduction à la philosophie esthétique. Paris : Payot, 313 p.

Simondon Gilbert (2005). L'individuation à la lumière des notions de forme et d'information. Grenoble : Jérôme Million (éd. orig. 1964), 571 p.

Soubeyran Olivier (2007). Pensée aménagiste et tautologies, in L'émergence des cosmo-politiques, sous la dir. de Jacques Lolive et d'Olivier Soubeyran. Paris : La Découverte, pp. 125-153.

Tissier Jean-Louis (1992). La géographie dans le prisme de l'environnement (1970-1990), in Du milieu à l'environnement. Pratiques et représentations du rapport homme/nature depuis la Renaissance ; sous la dir. de Marie-Claire Robic. Paris : Economica, pp. 201-236.

Tuan Yi-Fu (1976). Topophilia: a study of environmental perception, attitudes and values. Englewood Cliffs: Prentice Hall, 260 p. 